\title{
Necessary and Sufficient Conditions for a Hamiltonian with Discrete Eigenvalues to Have Time Operators
}

\author{
Asao Arai \\ Department of Mathematics, Hokkaido University \\ Sapporo, Hokkaido 060-0810 \\ Japan \\ E-mail: arai@math.sci.hokudai.ac.jp
}

August 22, 2008

\begin{abstract}
We present new classes of time operators of a Hamiltonian $H$ (a self-adjoint operator) with discrete eigenvalues which may be degenerate. Moreover we formulate necessary and sufficient conditions for $H$ to have time operators, determining the general form of them. As corollaries, non-existence theorems of time operators for some classes of $H$ are derived.
\end{abstract}

Mathematics Subject Classification (2000). 81Q10, 47N50.

Keywords. canonical commutation relation, Hamiltonian, time operator, eigenvalue.

\section{Introduction}

Let $\mathcal{H}$ be a complex Hilbert space and $H$ be a self-adjoint operator on $\mathcal{H}$. A symmetric operator $T$ on $\mathcal{H}$ is called a time operator of $H$ if there is a subspace $\mathcal{D} \neq\{0\}$ such that $\mathcal{D} \subset D(T H) \cap D(H T)$ (for a linear operator $A$ on $\mathcal{H}, D(A)$ denotes the domain of $A$ ) and the canonical commutation relation $(\mathrm{CCR})$ on $\mathcal{D}$

$$
[T, H] \psi=i \psi, \quad \forall \psi \in \mathcal{D}
$$

holds, where $[T, H]:=T H-H T$ and $i$ is the imaginary unit. The name "time operator" comes from the physical context where $H$ is the Hamiltonian of a quantum system (in that case, a canonical conjugate $T$ to $H$ is interpreted as an operator representing "time" in a suitable sense). But we use this terminology in the general mathematical context too. We call the subspace $\mathcal{D}$ a CCR-domain for the pair $(T, H)$. We do not assume that $\mathcal{D}$ is dense, since it is more natural and general, leaving possibility to have wider classes of time operators. 
From purely mathematical point of view, the pair $(T, H)$ is a (not necessarily selfadjoint) representation of the CCR with one degree of freedom. A study from this point of view has been made by Dorfmeister [7] in the case where $H$ is bounded.

There is a stronger version of time operator using the weak Weyl relation, a stronger form of the CCR ([1]-[5], [9]-[11]). But, in this paper, we do not discuss this type of time operators.

In the present paper we consider time operators of a self-adjoint operator $H$ whose spectrum "essentially" consists of discrete eigenvalues only (see Hypothesis (H) below and (2.5)), having in mind applications to the case where $H$ is a Hamiltonian in quantum theory. Such a time operator was proposed by Galapon [8] first. Then detailed, mathematically rigorous analysis on the Galapon time operator has been made by AraiMatsuzawa [6]. In the paper [6], however, considered was only the case where all the discrete eigenvalues of $H$ are simple with some growth condition. In the present paper, we do not assume the simplicity of the eigenvalues. We are interested in finding necessary and sufficient conditions for $H$ to have time operators as well as determining the general form of them. In this paper we solve this problem with respect to two classes of time operators. The first one is discussed in Section 2 and the second in Section 3. As corollaries of the main results on the problem, some non-existence theorems of time operators are established.

\section{Time Operators of a Hamiltonian with Discrete Eigenvalues (I)}

We denote the inner product and the norm of $\mathcal{H}$ by $\langle\cdot, \cdot\rangle$ (linear in the second variable) and $\|\cdot\|$ respectively.

Let $\mathbb{N}=\{1,2,3, \cdots\}$ be the set of natural numbers. A basic assumption in the present paper is as follows:

\section{Hypothesis ( $\mathbf{H})$}

The self-adjoint operator $H$ has a complete orthonormal system (CONS) $\left\{e_{n \alpha} \mid n \in\right.$ $\left.\mathbb{N}, \alpha=1, \cdots, M_{n}\right\} \subset \mathcal{H}$ of eigenvectors with discrete eigenvalues $\left\{E_{n}\right\}_{n \in \mathbb{N}}\left(E_{n} \neq\right.$ $\left.E_{m}, n \neq m, n, m \in \mathbb{N}\right)$ :

$$
\begin{aligned}
& H e_{n \alpha}=E_{n} e_{n \alpha}, \quad n \in \mathbb{N}, \alpha=1, \cdots, M_{n}, \\
& \left\langle e_{n \alpha}, e_{m \beta}\right\rangle=\delta_{n m} \delta_{\alpha \beta}, \quad \alpha=1, \cdots, M_{n}, \beta=1, \cdots, M_{m},
\end{aligned}
$$

where $\delta_{a b}$ is the Kronecker delta and $M_{n} \in \mathbb{N}$ is the multiplicity of eigenvalue $E_{n}$, obeying

$$
M_{n} \leq M_{n+1}, \quad n \in \mathbb{N} .
$$

We set

$$
M:=M_{1}
$$

Hypothesis $(\mathrm{H})$ implies that the spectrum of $H$, denoted $\sigma(H)$, is given by

$$
\sigma(H)=\overline{\left\{E_{n}\right\}_{n=1}^{\infty}},
$$


where the right hand side is the closure of the set $\left\{E_{n}\right\}_{n=1}^{\infty}$ and $\sigma(H) \backslash\left\{E_{n}\right\}_{n=1}^{\infty}$ contains no eigenvalues of $H$.

Remark 2.1 In the previous paper [6], only the case $M_{n}=1, \forall n \in \mathbb{N}$ (i.e., the case where each eigenvalue $E_{n}$ is simple) is considered. In the present paper we do not impose this condition on the multiplicities $M_{n}$.

The subspace

$$
\mathcal{D}_{0}:=\text { l.i.h. }\left\{e_{n \alpha} \mid n \in \mathbb{N}, \alpha=1, \cdots, M_{n}\right\}
$$

algebraically spanned by the vectors $e_{n \alpha}\left(n \in \mathbb{N}, \alpha=1, \cdots, M_{n}\right)$ is dense in $\mathcal{H}$.

\subsection{A general class of time operators of $H$}

Suppose that, for some $n_{0} \in \mathbb{N}$,

$$
\sum_{n=n_{0}}^{\infty} \frac{1}{E_{n}^{2}}<\infty
$$

Then, in the same way as in [6], one can define a linear operator $T_{0}$ as follows:

$$
\begin{aligned}
& D\left(T_{0}\right):=\mathcal{D}_{0}, \\
& T_{0} \psi:=i \sum_{n=1}^{\infty} \sum_{\alpha=1}^{M}\left(\sum_{m \neq n}^{\infty} \frac{\left\langle e_{m \alpha}, \psi\right\rangle}{E_{n}-E_{m}}\right) e_{n \alpha}, \quad \psi \in D\left(T_{0}\right) .
\end{aligned}
$$

It is easy to see that $T_{0}$ is a symmetric operator.

Remark 2.2 Under condition (2.6), $H$ is unbounded, since (2.6) implies that $\left|E_{n}\right| \rightarrow \infty$ as $n \rightarrow \infty$.

Remark 2.3 Galapon [8] proposed a time operator in the case where $M_{n}=M \geq 2$ for all $n \in \mathbb{N}$. In our notation, his time operator, denoted $\mathrm{T}_{M}$, is defined by

$$
\mathrm{T}_{M} \psi=i \sum_{n=1}^{\infty} \sum_{\alpha=1}^{M}\left(\sum_{m \neq n}^{\infty} \sum_{\beta \neq \alpha}^{M} \frac{\left\langle e_{m \beta}, \psi\right\rangle}{E_{n}-E_{m}}\right) e_{n \alpha}, \quad \psi \in D\left(\mathrm{~T}_{M}\right):=\mathcal{D}_{0} .
$$

It is asserted in [8] that $\mathrm{T}_{M}$ is a time operator of $H$ with a CCR-domain for $\left(\mathrm{T}_{M}, H\right)$ including the vectors $e_{n \alpha}-e_{m \alpha}, n, m \in \mathbb{N}, \alpha=1, \cdots, M$. But, unfortunately, this is false, because one has

$$
\left[\mathrm{T}_{M}, H\right]\left(e_{n \alpha}-e_{m \alpha}\right)=i \sum_{\beta \neq \alpha}^{M}\left(e_{n \beta}-e_{m \beta}\right)
$$

cf. the proof of Theorem 2.6 below. Besides this, the definition of $\mathrm{T}_{M}$ given by (2.9) is somewhat unnatural, because it does not cover the case $M=1$ as a special case. Our definition (2.8) is a generalization of the time operator in the case $M_{n}=1, \forall n \in \mathbb{N}[6,8]$.

Remark 2.4 In [8] and [6], it is assumed that $H$ is bounded below with $E_{n}<E_{n+1}, n \in$ $\mathbb{N}$. But, in the present paper, we do not assume the semi-boundedness (boundedness below or boundedness above). 
We introduce a subspace:

$$
\mathcal{E}_{M}:=\text { l.i.h. }\left\{e_{n \alpha}-e_{m \alpha} \mid n, m \in \mathbb{N}, \alpha=1, \cdots, M\right\} .
$$

This subspace is not necessarily dense in $\mathcal{H}$ :

Lemma 2.5 The subspace $\mathcal{E}_{M}$ is dense in $\mathcal{H}$ if and only if $M=M_{n}$ for all $n \in \mathbb{N}$.

Proof. We denote by $\mathcal{E}_{M}^{\perp}$ the orthogonal complement of $\mathcal{E}_{M}$. By a general theorem, $\mathcal{E}_{M}$ is dense in $\mathcal{H}$ if and only if $\mathcal{E}_{M}^{\perp}=\{0\}$.

(Necessity) Suppose that there is an $n_{0}$ such that $M_{n_{0}}>M$. Then

$$
\left\langle e_{n_{0}(M+1)}, e_{n \alpha}-e_{m \alpha}\right\rangle=0 \quad(n, m \in \mathbb{N}, \alpha=1, \cdots, M),
$$

which implies that $\mathcal{E}_{M}^{\perp} \neq\{0\}$. Hence $\mathcal{E}_{M}$ is not dense.

(Sufficiency) Suppose that $M=M_{n}$ for all $n \in \mathbb{N}$. Let $\psi \in \mathcal{E}_{M}^{\perp}$. Then $\left\langle e_{n \alpha}, \psi\right\rangle=$ $\left\langle e_{m \alpha}, \psi\right\rangle, n, m \in \mathbb{N}, \alpha=1, \cdots, M$. Hence, for all $N \in \mathbb{N}$,

$$
\sum_{n=1}^{N} \sum_{\alpha=1}^{M}\left|\left\langle e_{n \alpha}, \psi\right\rangle\right|^{2}=N \sum_{\alpha=1}^{M}\left|\left\langle e_{m \alpha}, \psi\right\rangle\right|^{2}
$$

Since $\left\{e_{n \alpha} \mid n \in \mathbb{N}, \alpha=1, \cdots, M\right\}$ is a CONS of $\mathcal{H}$ under the present assumption, the left hand side converges to $\|\psi\|^{2}$ as $N \rightarrow \infty$. Hence $\sum_{\alpha=1}^{M}\left|\left\langle e_{m \alpha}, \psi\right\rangle\right|^{2}=0$. Thus $\|\psi\|=0$, implying $\psi=0$.

The next theorem shows that $T_{0}$ is a time operator of $H$ with $\mathcal{E}_{M}$ being a CCR-domain for $\left(T_{0}, H\right)$ :

Theorem 2.6 Under assumptions (2.3) and (2.6), $\mathcal{E}_{M} \subset D\left(T_{0} H\right) \cap D\left(H T_{0}\right)$ and

$$
\left[T_{0}, H\right] \psi=i \psi, \quad \forall \psi \in \mathcal{E}_{M} .
$$

Proof. It is enough to show that, for all $n, m \in \mathbb{N}$ and $\alpha=1, \cdots, M$,

$$
\psi_{n m}:=e_{n \alpha}-e_{m \alpha}
$$

is in $D\left(T_{0} H\right) \cap D\left(H T_{0}\right)$ and (2.11) holds for $\psi=\psi_{n m}$. We have for all $n \in \mathbb{N}$

$$
T_{0} e_{n \alpha}=i \sum_{k \neq n}^{\infty} \frac{1}{E_{k}-E_{n}} e_{k \alpha}
$$

Hence

$$
T_{0} \psi_{n m}=i\left(E_{n}-E_{m}\right) \sum_{k \neq n, m}^{\infty} \frac{1}{\left(E_{k}-E_{n}\right)\left(E_{k}-E_{m}\right)} e_{k \alpha}+\frac{i}{E_{m}-E_{n}} e_{m \alpha}-\frac{i}{E_{n}-E_{m}} e_{n \alpha} .
$$

Since

$$
\sum_{k \neq n, m}^{\infty}\left|\frac{E_{k}}{\left(E_{k}-E_{n}\right)\left(E_{k}-E_{m}\right)}\right|^{2}<\infty
$$


by (2.6) and $H$ is closed, it follows that $T_{0} \psi_{n m} \in D(H)$ (i.e., $\psi_{n m} \in D\left(H T_{0}\right)$ ) and

$$
\begin{aligned}
H T_{0} \psi_{n m}= & i\left(E_{n}-E_{m}\right) \sum_{k \neq n, m}^{\infty} \frac{E_{k}}{\left(E_{k}-E_{n}\right)\left(E_{k}-E_{m}\right)} e_{k \alpha}+\frac{i E_{m}}{E_{m}-E_{n}} e_{m \alpha} \\
& -\frac{i E_{n}}{E_{n}-E_{m}} e_{n \alpha} .
\end{aligned}
$$

On the other hand, we have $H \psi_{n m}=E_{n} e_{n \alpha}-E_{m} e_{m \alpha}$. Hence $\psi_{n m} \in D\left(T_{0} H\right)$ and

$$
\begin{aligned}
T_{0} H \psi_{n, m}= & i E_{n} \sum_{k \neq n}^{\infty} \frac{1}{E_{k}-E_{n}} e_{k \alpha}-E_{m} i \sum_{k \neq m}^{\infty} \frac{1}{E_{k}-E_{m}} e_{k \alpha} \\
= & i\left(E_{n}-E_{m}\right) \sum_{k \neq n, m}^{\infty} \frac{E_{k}}{\left(E_{k}-E_{n}\right)\left(E_{k}-E_{m}\right)} e_{k \alpha}+i \frac{E_{n}}{E_{m}-E_{n}} e_{m \alpha} \\
& -i \frac{E_{m}}{E_{n}-E_{m}} e_{n \alpha} .
\end{aligned}
$$

Therefore we obtain

$$
T_{0} H \psi_{n m}-H T_{0} \psi_{n m}=i \psi_{n m}
$$

as desired.

Remark 2.7 It is easy to see that $\sum_{k \neq n}^{\infty} E_{k}^{2} /\left|E_{k}-E_{n}\right|^{2}=\infty$. Hence it follows from (2.12) that $T_{0} e_{n \alpha} \notin D(H)$. Therefore $\mathcal{D}_{0}$ is not a CCR-domain for $\left(T_{0}, H\right)$.

We next consider a perturbation of $T_{0}$ by a symmetric operator $T_{1}$ such that $T_{0}+T_{1}$ is a time operator of $H$.

Let $a:=\left\{a_{n}(\alpha, \beta) \mid n \in \mathbb{N}, \alpha, \beta=1, \cdots, M_{n}\right\}$ be a set of complex numbers such that

$$
a_{n}(\alpha, \beta)^{*}=a_{n}(\beta, \alpha), \quad n \in \mathbb{N}, \alpha, \beta=1, \cdots, M_{n},
$$

where $a_{n}(\alpha, \beta)^{*}$ is the complex conjugate of $a_{n}(\alpha, \beta)$. Then we define a linear operator $T_{1}(a)$ on $\mathcal{H}$ as follows:

$$
\begin{aligned}
& D\left(T_{1}(a)\right):=\left\{\left.\psi \in \mathcal{H}\left|\sum_{n=1}^{\infty} \sum_{\alpha=1}^{M_{n}}\right| \sum_{\beta=1}^{M_{n}} a_{n}(\alpha, \beta)\left\langle e_{n \beta}, \psi\right\rangle\right|^{2}<\infty\right\}, \\
& T_{1}(a) \psi:=\sum_{n=1}^{\infty} \sum_{\alpha=1}^{M_{n}}\left(\sum_{\beta=1}^{M_{n}} a_{n}(\alpha, \beta)\left\langle e_{n \beta}, \psi\right\rangle\right) e_{n \alpha}, \quad \psi \in D\left(T_{1}(a)\right) .
\end{aligned}
$$

It follows that

$$
\mathcal{D}_{0} \subset D\left(T_{1}(a)\right)
$$

with

$$
T_{1}(a) e_{n \alpha}=\sum_{\beta=1}^{M_{n}} a_{n}(\beta, \alpha) e_{n \beta}, \quad \forall n \in \mathbb{N}, \alpha=1, \cdots, M_{n}
$$


It is easy to see that $T_{1}(a)$ is a symmetric operator.

Using (2.16), we see that $\mathcal{D}_{0} \subset D\left(H T_{1}(a)\right) \cap D\left(T_{1}(a) H\right)$ and

$$
T_{1}(a) H \psi=H T_{1}(a) \psi, \quad \forall \psi \in \mathcal{D}_{0} .
$$

By this fact and Theorem 2.6 we obtain the next theorem:

Theorem 2.8 Assume (2.3) and (2.6). Let a be as above and

$$
T(a):=T_{0}+T_{1}(a) .
$$

Then $T(a)$ is a time operator of $H$ with $\mathcal{E}_{M}$ being a $C C R$-domain for $(T(a), H)$.

Thus (2.6) gives a sufficient condition for $H$ with Hypothesis $(H)$ and $(2.3)$ to have time operators of the form (2.17).

Remark 2.9 Boundedness or unboundedness of $T(a)$ can be investigated in the same way as in [6]. But, here, we do not go into the details.

\subsection{Necessary condition for $H$ to have time operators and the general form of them}

We are now ready to derive a necessary condition for $H$ to have time operators and their general form.

Theorem 2.10 Let $H$ be a self-adjoint operator satisfying Hypothesis (H) and (2.3), and $T$ be a time operator of $H$ such that $\mathcal{E}_{M}$ is a CCR-domain for $(T, H)$ and $e_{n \alpha} \in D(T), \forall n \in$ $\mathbb{N}, \alpha=1, \cdots, M$. Then $H$ is unbounded and there is an $n_{0} \in \mathbb{N}$ such that (2.6) holds.

Moreover, the following (i) and (ii) hold:

(i) Let $M=M_{n}, \forall n \in \mathbb{N}$. Then, for all $\psi \in D(T)$,

$$
\sum_{n=1}^{\infty} \sum_{\alpha=1}^{M}\left|i \sum_{m \neq n}^{\infty} \frac{\left\langle e_{m \alpha}, \psi\right\rangle}{E_{n}-E_{m}}+\sum_{\beta=1}^{M}\left\langle e_{n \alpha}, T e_{n \beta}\right\rangle\left\langle e_{n \beta}, \psi\right\rangle\right|^{2}<\infty
$$

and

$$
T \psi=\sum_{n=1}^{\infty} \sum_{\alpha=1}^{M}\left(i \sum_{m \neq n}^{\infty} \frac{\left\langle e_{m \alpha}, \psi\right\rangle}{E_{n}-E_{m}}+\sum_{\beta=1}^{M}\left\langle e_{n \alpha}, T e_{n \beta}\right\rangle\left\langle e_{n \beta}, \psi\right\rangle\right) e_{n \alpha} .
$$

In particular, one has

$$
T=T(a(T))=T_{0}+T_{1}(a(T)) \quad \text { on } \mathcal{D}_{0},
$$

where

$$
a(T)_{n}(\alpha, \beta):=\left\langle e_{n \alpha}, T e_{n \beta}\right\rangle, \quad n \in \mathbb{N}, \alpha, \beta=1, \cdots, M .
$$


(ii) Let $k$ be a natural number such that $M=M_{n}, n=1, \cdots, k$, and $M_{n}>M, n \geq$ $k+1$. Then, for all $\psi \in D(T)$,

$$
\begin{aligned}
& \sum_{n=1}^{\infty} \sum_{\alpha=1}^{M}\left|i \sum_{m \neq n}^{\infty} \frac{\left\langle e_{m \alpha}, \psi\right\rangle}{E_{n}-E_{m}}+\sum_{\beta=1}^{M_{n}}\left\langle e_{n \alpha}, T e_{n \beta}\right\rangle\left\langle e_{n \beta}, \psi\right\rangle\right|^{2}<\infty \\
& \sum_{n=k+1}^{\infty} \sum_{\alpha=M+1}^{M_{n}}\left|\left\langle e_{n \alpha}, T \psi\right\rangle\right|^{2}<\infty
\end{aligned}
$$

and

$$
T \psi=\sum_{n=1}^{\infty} \sum_{\alpha=1}^{M}\left(i \sum_{m \neq n}^{\infty} \frac{\left\langle e_{m \alpha}, \psi\right\rangle}{E_{n}-E_{m}}+\sum_{\beta=1}^{M_{n}}\left\langle e_{n \alpha}, T e_{n \beta}\right\rangle\left\langle e_{n \beta}, \psi\right\rangle\right) e_{n \alpha}+S_{T} \psi
$$

where

$$
S_{T} \psi:=\sum_{n=k+1}^{\infty} \sum_{\alpha=M+1}^{M_{n}}\left\langle e_{n \alpha}, T \psi\right\rangle e_{n \alpha}
$$

with

$$
D\left(S_{T}\right):=\left\{\left.\psi \in \mathcal{H}\left|\sum_{n=k+1}^{\infty} \sum_{\alpha=M+1}^{M_{n}}\right|\left\langle e_{n \alpha}, T \psi\right\rangle\right|^{2}<\infty\right\} .
$$

Proof. By (1.1) with $\mathcal{D}=\mathcal{E}_{M}$ and (2.1), we have

$$
E_{n} T e_{n \alpha}-E_{m} T e_{m \alpha}-H\left(T e_{n \alpha}-T e_{m \alpha}\right)=i\left(e_{n \alpha}-e_{m \alpha}\right), \quad n, m \in \mathbb{N}, \alpha=1, \cdots, M .
$$

Let $n \neq m$ and take the inner product of the both sides with $e_{m \beta}, \beta=1, \cdots, M_{m}$. Then, by the symmetry of $H$ and (2.1), we have

$$
\left\langle e_{m \beta}, T e_{n \alpha}\right\rangle=\frac{i}{E_{m}-E_{n}} \delta_{\alpha \beta} .
$$

Since $\left\{e_{m \beta} \mid m \in \mathbb{N}, \beta=1, \cdots, M_{m}\right\}$ is a CONS of $\mathcal{H}$, it follows from the Parseval equality that $\left\|T e_{n \alpha}\right\|^{2}=\sum_{m=1}^{\infty} \sum_{\beta=1}^{M_{m}}\left|\left\langle e_{m \beta}, T e_{n \alpha}\right\rangle\right|^{2}$, implying that

$$
\sum_{m \neq n}^{\infty} \frac{1}{\left|E_{m}-E_{n}\right|^{2}}<\infty
$$

with

$$
\left\|T e_{n \alpha}\right\|^{2}=\sum_{m \neq n}^{\infty} \frac{1}{\left|E_{m}-E_{n}\right|^{2}}+\sum_{\beta=1}^{M_{n}}\left|\left\langle e_{n \beta}, T e_{n \alpha}\right\rangle\right|^{2}
$$

and

$$
T e_{n \alpha}=\sum_{m \neq n}^{\infty} \frac{i}{E_{m}-E_{n}} e_{m \alpha}+\sum_{\beta=1}^{M_{n}}\left\langle e_{n \beta}, T e_{n \alpha}\right\rangle e_{n \beta}
$$


Property (2.24) is equivalent to (2.6) and hence $\left|E_{m}\right| \rightarrow \infty$ as $m \rightarrow \infty$. Hence $H$ is unbounded. Thus the first half of the theorem is proved.

Next we prove the latter half of the theorem.

(i) Let $M_{n}=M, \forall n \in \mathbb{N}$. Then $\left\{e_{n \alpha} \mid n \in \mathbb{N}, \alpha=1, \cdots, M\right\}$ is a CONS of $\mathcal{H}$. Hence, each vector $\psi \in D(T)$, we have by the Parseval equality

$$
\|T \psi\|^{2}=\sum_{n=1}^{\infty} \sum_{\alpha=1}^{M}\left|\left\langle e_{n \alpha}, T \psi\right\rangle\right|^{2}=\sum_{n=1}^{\infty} \sum_{\alpha=1}^{M}\left|\left\langle T e_{n \alpha}, \psi\right\rangle\right|^{2}
$$

with the expansion

$$
T \psi=\sum_{n=1}^{\infty} \sum_{\alpha=1}^{M}\left\langle e_{n \alpha}, T \psi\right\rangle e_{n \alpha}=\sum_{n=1}^{\infty} \sum_{\alpha=1}^{M}\left\langle T e_{n \alpha}, \psi\right\rangle e_{n \alpha} .
$$

Then, by (2.25), we have (2.18) and (2.19).

(ii) Under the present condition, $\left\{e_{n \alpha} \mid n \in \mathbb{N}, \alpha=1, \cdots, M\right\} \cup\left\{e_{n \beta} \mid n \geq k+1, \beta=\right.$ $\left.M+1, \cdots, M_{n}\right\}$ is a CONS of $\mathcal{H}$. Hence, for all $\psi \in D(T)$, we have by the Parseval equality

$$
\|T \psi\|^{2}=\sum_{n=1}^{\infty} \sum_{\alpha=1}^{M}\left|\left\langle e_{n \alpha}, T \psi\right\rangle\right|^{2}+\sum_{n=k+1}^{\infty} \sum_{\alpha=M+1}^{M_{n}}\left|\left\langle e_{n \alpha}, T \psi\right\rangle\right|^{2} .
$$

Hence (2.21) and (2.22) follow. Also we have for all $\psi \in D(T)$

$$
T \psi=\sum_{n=1}^{\infty} \sum_{\alpha=1}^{M}\left\langle e_{n \alpha}, T \psi\right\rangle e_{n \alpha}+\sum_{n=k+1}^{\infty} \sum_{\alpha=M+1}^{M_{n}}\left\langle e_{n \alpha}, T \psi\right\rangle e_{n \alpha} .
$$

The first term on the right hand side is of the same form as that in part (i). The second term is equal to $S_{T} \psi$.

Remark 2.11 Consider the case where at least one of $E_{n}$ 's is degenerate. Let $\mathcal{D}$ be a subspace including $\left\{e_{n \alpha}-e_{n \beta} \mid n \in \mathbb{N}, \alpha, \beta=1, \cdots, M_{n}\right\}$. Then there exist no time operators $T$ of $H$ such that $\mathcal{D}_{0} \subset D(T)$ and $\mathcal{D}$ is a $C C R$-domain for $(T, H)$.

Indeed, suppose that there existed such a time operator $T$ of $H$. Let $E_{n_{0}}$ be a degenerate eigenvalue of $H: M_{n_{0}} \geq 2$. Then, putting $\psi=e_{n_{0} \alpha}-e_{n_{0} \beta}\left(\alpha \neq \beta, \alpha, \beta=1, \cdots, M_{n_{0}}\right)$ in (1.1), we have $E_{n_{0}}\left(T e_{n_{0} \alpha}-T e_{n_{0} \beta}\right)-H\left(T e_{n_{0} \alpha}-T e_{n_{0} \beta}\right)=i\left(e_{n_{0} \alpha}-e_{n_{0} \beta}\right)$. Taking the inner product of the both sides with $e_{n_{0} \alpha}$, we have $0=i$, which is a contradiction.

\subsection{Non-existence theorems of time operators}

Theorem 2.10 can be read as non-existence theorems of time operators for a class of $H$ as shown below.

Theorem 2.12 Let $H$ be a self-adjoint operator with Hypothesis (H) and (2.3) such that

$$
\sum_{n=n_{0}}^{\infty} \frac{1}{E_{n}^{2}}=\infty
$$

for some $n_{0} \in \mathbb{N}$. Then there exist no time operators $T$ of $H$ such that $\mathcal{D}_{0} \subset D(T)$ and $\mathcal{E}_{M}$ is a CCR-domain for $(T, H)$. 
Proof. This follows from the contraposition of Theorem 2.10.

A simple consequence of this theorem is given as follows:

Theorem 2.13 Let $H$ be a self-adjoint operator with Hypothesis (H) and (2.3). Suppose that there exist a constant $\alpha \in[0,1 / 2]$ and a real bounded sequence $\left\{b_{n}\right\}_{n=1}^{\infty}$ satisfying

$$
E_{n}=b_{n} n^{\alpha}+o\left(n^{\alpha}\right) \quad(n \rightarrow \infty) .
$$

Then there exist no time operators $T$ of $H$ such that $\mathcal{D}_{0} \subset D(T)$ and $\mathcal{E}_{M}$ is a CCR-domain for $(T, H)$.

Proof. Let $b:=\sup _{n \in \mathbb{N}}\left|b_{n}\right|<\infty$. Then, by (2.27) and Hypothesis (H), there are constants $n_{0} \in \mathbb{N}$ and $b^{\prime}>0$ such that

$$
0<\left|E_{n}\right| \leq\left(b+b^{\prime}\right) n^{\alpha}, \quad n \geq n_{0} .
$$

Hence

$$
\sum_{n=n_{0}}^{\infty} \frac{1}{E_{n}^{2}} \geq \frac{1}{\left(b+b^{\prime}\right)^{2}} \sum_{n=n_{0}}^{\infty} \frac{1}{n^{2 \alpha}}=\infty .
$$

Therefore (2.26) holds. Thus the desired result follows.

Theorem 2.14 Let $H$ be a bounded self-adjoint operator with Hypothesis (H) and (2.3). Then there exist no time operators $T$ of $H$ such that $\mathcal{D}_{0} \subset D(T)$ and $\mathcal{E}_{M}$ is a CCR-domain for $(T, H)$.

Proof. If $H$ is bounded, then the sequence $\left\{E_{n}\right\}_{n=1}^{\infty}$ is bounded. Hence this is the case where $\alpha=0$ in (2.27). Thus Theorem 2.13 implies the desired result.

\section{Time Operators of a Hamiltonian with Discrete Eigenvalues (II)}

In this section we present another type of time operators of $H$. Here we do not assume (2.3).

We define

$$
\bar{e}_{n}:=\frac{1}{\sqrt{M_{n}}} \sum_{\alpha=1}^{M_{n}} e_{n \alpha} .
$$

Then

$$
H \bar{e}_{n}=E_{n} \bar{e}_{n}, \quad n \in \mathbb{N}
$$

and $\left\{\bar{e}_{n}\right\}_{n=1}^{\infty}$ is an orthonormal system of $\mathcal{H}:\left\langle\bar{e}_{n}, \bar{e}_{m}\right\rangle=\delta_{n m}, n, m \in \mathbb{N}$.

We introduce a subspace:

$$
\mathcal{F}_{0}:=\text { l.i.h. }\left\{\bar{e}_{n} \mid n \in \mathbb{N}\right\} \text {. }
$$


It is easy to see that $\mathcal{F}_{0}$ is dense if and only if $M_{n}=1$ for all $n \in \mathbb{N}$.

Assume (2.6). Then, as in the case of the operator $T_{0}$ in Section 2, one can define a linear operator $\widehat{T}_{0}$ on $\mathcal{H}$ as follows:

$$
\begin{aligned}
& D\left(\widehat{T}_{0}\right):=\left\{\psi=\psi_{1}+\psi_{2} \mid \psi_{1} \in \mathcal{F}_{0}, \psi_{2} \in \mathcal{F}_{0}^{\perp}\right\} \\
& \widehat{T}_{0} \psi:=i \sum_{n=1}^{\infty}\left(\sum_{m \neq n}^{\infty} \frac{\left\langle\bar{e}_{m}, \psi\right\rangle}{E_{n}-E_{m}}\right) \bar{e}_{n}, \quad \psi \in D\left(\widehat{T}_{0}\right),
\end{aligned}
$$

It is easy to see that $\widehat{T}_{0}$ is densely defined and symmetric.

We remark that, if $M_{n}=1, \forall n \in \mathbb{N}$, then

$$
\widehat{T}_{0}=T_{0} .
$$

Let

$$
\mathcal{F}_{-}:=\text {l.i.h. }\left\{\bar{e}_{n}-\bar{e}_{m} \mid n, m \in \mathbb{N}\right\} \text {. }
$$

It is obvious that

$$
\mathcal{F}_{-} \subset \mathcal{F}_{0} .
$$

It is shown that, if every $E_{n}$ is simple, then $\mathcal{F}_{-}$is dense in $\mathcal{H}[6,8]$. But $\mathcal{F}_{-}$is not dense if at least one of $E_{n}(n \in \mathbb{N})$ is degenerate.

Theorem 3.1 The operator $\widehat{T}_{0}$ is a time operator of $H$ with $\mathcal{F}_{-}$being a CCR-domain for $\left(\widehat{T}_{0}, H\right)$. Namely

$$
\mathcal{F}_{-} \subset D\left(\widehat{T}_{0} H\right) \cap D\left(H \widehat{T}_{0}\right)
$$

and

$$
\left[\widehat{T}_{0}, H\right] \psi=i \psi, \quad \forall \psi \in \mathcal{F}_{-} .
$$

Proof. Let $\bar{\psi}_{n m}:=\bar{e}_{n}-\bar{e}_{m}$. Then, in quite the same way as in the proof of Theorem 2.6, one can show that $\bar{\psi}_{n m} \in D\left(\widehat{T}_{0} H\right) \cap D\left(H \widehat{T}_{0}\right)$ and

$$
\widehat{T}_{0} H \bar{\psi}_{n m}-H \widehat{T}_{0} \bar{\psi}_{n m}=i \bar{\psi}_{n m} .
$$

Thus the desired result follows.

For a real sequence $c=\left\{c_{n}\right\}_{n=1}^{\infty}$, we define a linear operaotr $S(c)$ on $\mathcal{H}$ as follows:

$$
\begin{aligned}
& D(S(c)):=\left\{\left.\psi \in \mathcal{H}\left|\sum_{n=1}^{\infty}\right| c_{n}\right|^{2}\left|\left\langle\bar{e}_{n}, \psi\right\rangle\right|^{2}<\infty\right\}, \\
& S(c) \psi:=\sum_{n=1}^{\infty} c_{n}\left\langle\bar{e}_{n}, \psi\right\rangle \bar{e}_{n}, \quad \psi \in D(S(c)) .
\end{aligned}
$$

Obviously we have

$$
\mathcal{F}_{0} \subset D(S(c))
$$

with

$$
S(c) \bar{e}_{n}=c_{n} \bar{e}_{n}, \quad \forall n \in \mathbb{N} .
$$


Hence $c_{n}$ is an eigenvalue of $S(c)$. It follows that $S(c)$ is a symmetric operator. Using (3.8), we see that $\mathcal{F}_{0} \subset D(H S(c)) \cap D(S(c) H)$ and

$$
S(c) H \psi=H S(c) \psi, \quad \forall \psi \in \mathcal{F}_{0} .
$$

Thus the operator

$$
\widehat{T}(c):=\widehat{T}_{0}+S(c)
$$

is a time operator of $H$ with $\mathcal{F}_{-}$being a CCR-domain for $(\widehat{T}(c), H)$.

Let

$$
\mathcal{F}:=\overline{\mathcal{F}}_{0} \text {. }
$$

We denote the range of $T$ by $\operatorname{Ran}(T)$.

Theorem 3.2 Let $H$ be a self-adjoint operator satisfying Hypothesis (H) and $T$ be a time operator of $H$ such that $\mathcal{F}_{0} \subset D(T), \operatorname{Ran}(T) \subset \mathcal{F}$ and $\mathcal{F}_{-}$is a CCR-domain for $(T, H)$. Then $H$ is unbounded and there is an $n_{0} \in \mathbb{N}$ such that (2.6) holds.

Moreover, for all $\psi \in D(T)$,

$$
\sum_{n=1}^{\infty}\left|i \sum_{m \neq n}^{\infty} \frac{\left\langle\bar{e}_{m}, \psi\right\rangle}{E_{n}-E_{m}}+\left\langle\bar{e}_{n}, T \bar{e}_{n}\right\rangle\left\langle\bar{e}_{n}, \psi\right\rangle\right|^{2}<\infty
$$

and

$$
T \psi=\sum_{n=1}^{\infty}\left(i \sum_{m \neq n}^{\infty} \frac{\left\langle\bar{e}_{m}, \psi\right\rangle}{E_{n}-E_{m}}+\left\langle\bar{e}_{n}, T \bar{e}_{n}\right\rangle\left\langle\bar{e}_{n}, \psi\right\rangle\right) \bar{e}_{n} .
$$

In particular, one has

$$
T=\widehat{T}(c(T))=\widehat{T}_{0}+S(c(T)) \quad \text { on } \mathcal{F}_{0},
$$

where

$$
c(T):=\left\{\left\langle\bar{e}_{n}, T \bar{e}_{n}\right\rangle\right\}_{n=1}^{\infty} .
$$

Proof. By (1.1) with $\mathcal{D}=\mathcal{F}_{-}$and (3.1), we have

$$
E_{n} T \bar{e}_{n}-E_{m} T \bar{e}_{m}-H\left(T \bar{e}_{n}-T \bar{e}_{m}\right)=i\left(\bar{e}_{n}-\bar{e}_{m}\right), \quad n, m \in \mathbb{N} .
$$

Let $n \neq m$ and take the inner product of the both sides with $\bar{e}_{m}$. Then we obtain

$$
\left\langle\bar{e}_{m}, T \bar{e}_{n}\right\rangle=\frac{i}{E_{m}-E_{n}} .
$$

Since $\left\{\bar{e}_{m}\right\}_{m=1}^{\infty}$ is an orthonormal system of $\mathcal{H}$ and $\operatorname{Ran}(T) \subset \mathcal{F}$, it follows from the Parseval equality that $\left\|T \bar{e}_{n}\right\|^{2}=\sum_{m=1}^{\infty}\left|\left\langle\bar{e}_{m}, T \bar{e}_{n}\right\rangle\right|^{2}$, implying (2.24) with

$$
\left\|T \bar{e}_{n}\right\|^{2}=\sum_{m \neq n}^{\infty} \frac{1}{\left|E_{m}-E_{n}\right|^{2}}+\left|\left\langle\bar{e}_{n}, T \bar{e}_{n}\right\rangle\right|^{2}
$$


and

$$
T \bar{e}_{n}=\sum_{m \neq n}^{\infty} \frac{i}{E_{m}-E_{n}} \bar{e}_{m}+\left\langle\bar{e}_{n}, T \bar{e}_{n}\right\rangle \bar{e}_{n} .
$$

Hence (2.6) holds. In particular, $\left|E_{m}\right| \rightarrow \infty$ as $m \rightarrow \infty$. Hence $H$ is unbounded.

By condition $\operatorname{Ran}(T) \subset \mathcal{F}$ and the Parseval equality again, we have for all $\psi \in D(T)$, $\|T \psi\|^{2}=\sum_{n=1}^{\infty}\left|\left\langle\bar{e}_{n}, T \psi\right\rangle\right|^{2}$ and

$$
T \psi=\sum_{n=1}^{\infty}\left\langle\bar{e}_{n}, T \psi\right\rangle \bar{e}_{n}=\sum_{n=1}^{\infty}\left\langle T \bar{e}_{n}, \psi\right\rangle \bar{e}_{n} .
$$

Then, by (3.14), we have (3.11) and (3.12).

As in Theorem 2.10, Theorem 3.2 can be read as non-existence theorems of time operators for a class of $H$.

Theorem 3.3 Let $H$ be a self-adjoint operator with Hypothesis (H) such that (2.26) holds for some $n_{0} \geq 1$. Then there exist no time operators $T$ of $H$ such that $\mathcal{F}_{0} \subset D(T)$, $\operatorname{Ran}(T) \in \mathcal{F}$ and $\mathcal{F}_{-}$is a $C C R$-domain for $(T, H)$.

Proof. This follows from the contraposition of Theorem 3.2.

Theorem 3.4 Let $H$ be a self-adjoint operator with Hypothesis (H). Suppose that there exist a constant $\alpha \in[0,1 / 2]$ and a real bounded sequence $\left\{b_{n}\right\}_{n=1}^{\infty}$ such that (2.27) holds. Then there exist no time operators $T$ of $H$ such that $\mathcal{F}_{0} \subset D(T), \operatorname{Ran}(T) \subset \mathcal{F}$ and $\mathcal{F}_{-}$is a CCR-domain for $(T, H)$.

Proof. Similar to the proof of Theorem 2.13.

Theorem 3.5 Let $H$ be a bounded self-adjoint operator with Hypothesis (H). Then there exist no time operators $T$ of $H$ such that $\mathcal{F}_{0} \subset D(T), \operatorname{Ran}(T) \subset \mathcal{F}$ and $\mathcal{F}_{-}$is a $C C R$ domain for $(T, H)$.

Proof. Similar to the proof of Theorem 2.14

\section{References}

[1] A. Arai, Generalized weak Weyl relation and decay of quantum dynamics, Rev. Math. Phys. 17 (2005) , 1071-1109 .

[2] A. Arai, Spectrum of time operators, Lett. Math. Phys. 80 (2007), 211-221.

[3] A. Arai, Some aspects of time operators, in Quantum Bio-Informatics (Editors: L. Accardi, W. Freudenberg and M. Ohya), World Scientific, Singapore, 2008, 26-35.

[4] A. Arai, On the uniqueness of weak Weyl representations of the canonical commutation relation, Lett. Math. Phys. 85 (2008), 15-25. 
[5] A. Arai and Y. Matsuzawa, Construction of a Weyl representation from a weak Weyl representation of the canonical commutation relation, Lett. Math. Phys. 83 (2008), 201-211.

[6] A. Arai and Y. Matsuzawa, Time operators of a Hamiltonian with purely discrete spectrum, mp_arc 08-24, to be published in Rev. Math. Phys.

[7] G. Dorfmeister and J. Dorfmeister, Classification of certain pairs of operators $(P, Q)$ satisfying $[P, Q]=-i \mathrm{Id}$, J. Funct. Anal. 57 (1984), 301-328.

[8] E. A. Galapon, Self-adjoint time operator is the rule for discrete semi-bounded Hamiltonians, Proc. R. Soc. Lond. A 458 (2002), 2671-2689.

[9] M. Miyamoto, A generalized Weyl relation approach to the time operator and its connection to the survival probability, J. Math. Phys. 42 (2001), 1038-1052. 104, $570-578$.

[10] K. Schmüdgen, On the Heisenberg commutation relation. I, J. Funct. Anal. 50 (1983), 8-49.

[11] K. Schmüdgen, On the Heisenberg commutation relation. II, Publ. RIMS, Kyoto Univ. 19 (1983), 601-671. 